\title{
Sex Differences in Stress Reactivity to the Trier Social Stress Test in Virtual Reality
}

This article was published in the following Dove Press journal:

Psychology Research and Behavior Management

\author{
Qing Liu (D) ${ }^{1,2}$ \\ Wenjuan Zhang ${ }^{3}$ \\ 'College of Education and Technology, \\ Zhejiang University of Technology, \\ Hangzhou, People's Republic of China; \\ ${ }^{2}$ School of Psychology, Beijing Normal \\ University, Beijing, People's Republic of \\ China; ${ }^{3}$ Mental Health Education Center, \\ Xidian University, Xi'an, Shaanxi, People's \\ Republic of China
}

Objective: The aims of the present study were twofold: 1) to examine the effects of a virtual reality version of Trier Social Stress Test (TSST-VR) using a placebo as the control condition; 2) to delineate sex differences in psychophysiological responses following the TSSTVR.

Methods: Healthy young male $(n=30)$ and female $(n=30)$ undergraduates were randomly assigned to a psychosocial stress protocol condition or to a non-stressful control condition (placebo) also under virtual reality environment (VR). Electrodermal activity (EDA), heart rate $(\mathrm{HR})$ and heart rate variability (HRV) were measured throughout the condition. The visual analog scale (VAS) was used to assess the perceived stress before and after the condition. We also included subjective scales of emotional states and coping.

Results: Different ANOVAs showed that after VR, the stress group reported higher scores on VAS than the non-stress group. Before VR, compared with females, the males showed stronger EDA and higher HRV. Under VR, the males had lower HR. After VR, the males' HR was still lower than females', but their HRV was higher than females'. Finally, the correlation between subjective and objective reactivity demonstrated that HRV during the experiment was negatively correlated to depression and negative affect. The HRV after VR was negatively correlated to the positive coping but was positively correlated to the depression.

Conclusion: These findings suggest that the TSST-VR could be used as an available tool for testing sex differences to psychosocial stress induction in experimental settings. Compared with females, males were more sensitive to stress. The scores on depression, negative affect and positive coping before the stress induction may be able to predict the arousal of the sympathetic nervous system across the stress situations.

Keywords: Trier Social Stress Test, virtual reality, placebo version, sex difference, stress reactivity, visual analog scale

\section{Introduction}

Stress has been defined as "experiences that cause feelings of anxiety and frustration because they push us beyond our ability to successfully cope". ${ }^{1}$ The effective induction of a stressor and measured stress reactivity have been important to stress research, and this has both theoretical and practical implications. ${ }^{2,3}$ Researchers have explored many means of inducing acute stress reactivity in experimental settings, and the Trier Social Stress Test (TSST) has been one of the most popular methods because its validity has been shown with both ordinary participants and clinical samples. ${ }^{4,5}$ In brief, the TSST requests the participants to give a speech and perform a math task in front of committee comprising professionally trained actors
Correspondence: Qing Liu

Zhejiang University of Technology

Hangzhou, 310014, People's Republic of China

Tel/Fax +86 57I 85290II4

Email 0616566@zju.edu.cn 
who do not express any emotional variation during the experiment. The participants are thus nervous because of social evaluation and uncontrollable factors. ${ }^{6}$

One of the disadvantages of the TSST is the difficulty of maintaining the constancy of the test conditions, especially requesting the experimenter to maintain the identical demeanor for all the participants. ${ }^{7,8}$ The TSST in a virtual reality environment (TSST-VR) uses the virtual persons as experimenters who give unbiased responses to all the participants. ${ }^{9,10}$ The virtual reality of a committee could greatly reduce the practical problems, labor and costs required of the TSST committee. ${ }^{11}$ Moreover, the use of VR provides a measure of human interaction with a dynamic and realist 3D environment, which facilitates the use of instruments for psychophysiological evaluation. ${ }^{12,13}$ Furthermore, the use of the TSST to induce participants' stress reactivity in the laboratory requires a control (nonstress) condition. The purpose of the control design was to demonstrate that the stress reactivity observed in the experiment was caused by the social evaluation and uncontrollable factors of the TSST rather than by other factors such as the physical or cognitive demands of the task itself. ${ }^{14}$ Het et al, ${ }^{15}$ believed that the control condition that comprised a simple talk and a simple calculation was the most appropriate way to test the effects of psychosocial stressors on the individual. They named this the "Placebo Version" of the TSST. In order to devise a TSST protocol that reduces the variability of procedure while maintaining the social-evaluative and uncontrollable nature of the task, the TSST-VR should use the placebo as its control condition.

As a psychosocial stressor, the TSST induces the arousal of the hypothalamic-pituitary- adrenal (HPA) axis, usually measured by cortical, and sympathetic nervous system (SNS), measured by electrodermal activity (EDA), heart rate (HR), and heart rate variability (HRV). ${ }^{16}$ The EDA, HR and HRV can be continuously measured throughout the TSST which conferred them with an advantage over measures which were less practical to take during the test. ${ }^{17}$ Therefore, the HRVs (especially the spectral indices) have been found related to stress by many studies. ${ }^{18,19}$ Specifically, the spectral indices of HRVs included the high frequency of HRV (HF; $0.15-0.40 \mathrm{~Hz}$ ), the low frequency of HRV (LF; 0.04-0.15 Hz), and the ratio of $\mathrm{LF}$ and $\mathrm{HF}^{20}$ In addition to physiological reactivity, the TSST will also induce psychological reactivity such as stress perception, anxiety, and the insecurity affect. $^{21,22}$
While the effectiveness of TSST in inducing physiological and psychological changes is clear, research has revealed that there are considerable intra- and interindividual variations in the psychobiological stress responses elicited. ${ }^{23}$ In various studies, the self-report and physiological and hormonal responses to the TSST, differed between men and women. ${ }^{24}$ Traditional TSST studies have shown that this task results in larger increases of salivary cortisol in men than in women. ${ }^{25,26}$ Salivary cortisol is an output of the HPA axis, which can be influenced by many interactive systems and hormones within the body, including androgen, testosterone, and estrogen. ${ }^{27}$ These studies indicated that the sex differences in salivary cortisol responses, can be influenced by factors related to both interindividual (ie, cortisol and biological sex), and intraindividual (ie, cortisol, menstrual cycle and oral contraceptives) variability. ${ }^{28,29}$ Therefore, when exploring the sex differences induced by TSST, attention should be drawn to the importance of procedural variations, and the extent to which they can amplify or negate sex differences in salivary cortisol output.

In addition, a recent meta-analysis of cortisol reactivity to TSST-VR, found participants who were under 25 years old, or all male, showed greater effect sizes for cortisol reactivity. ${ }^{30}$ This may explain why the few studies that focused on sex differences in stress response patterns in TSST-VR, showed no differences in HR and cortisol between men and women. ${ }^{31,32}$ Specifically, MonteroLópez et $\mathrm{al}^{31}{ }^{31}$ when using the TSST-VR, only obtained distinct stress perceptions rather than responses (cortisol or HR) in men (ie, higher realism when TSST-VR was presented on a screen), and women (ie, more involvement in immersion of VR). Subsequently, they conducted further research on the relationship between women's menstrual cycle and stress-invoked cortisol secretion, and found that the menstrual cycle phase tended to influence cortisol response to TSST-VR. ${ }^{33}$ Similar results were observed by Santl et al, ${ }^{32}$ who found significant differences only for stress ratings. In their studies, women who took oral contraceptives, claimed significantly higher stress levels than men. The reasons for these differences in stress perception are unknown, but are likely to be related to both hormonal influences (ie, menstrual cycle and use of hormonal contraceptives) $^{34}$ and methodological aspects (ie, stress marker recording). ${ }^{35}$

With the aim of identifying "sex-specific" stress reactivity indices, and determining which part of the TSST induced a greater deal of stress in the participant, Juster 
et $\mathrm{al}^{36}$ found that markers of stress, which can be measured throughout the TSST-VR, would respond in a more time-locked manner than cortisol (ie, EDA and HR) and help in exploring sex differences of stress reactivity under psychosocial stressors.

The aims of the present study were twofold: 1) to examine the effects of a virtual reality version of Trier Social Stress Test (TSST-VR) using a placebo as its control condition; 2) to delineate sex differences in psychophysiological responses following the TSST-VR. Based on the above reviews, we hypothesized that the stress reactivity produced by the TSST-VR would be different in men and women. Compared to women, men would be more sensitive to stress. We further hypothesized that the subjective and objective reactivity to stress would be correlated across the experiment.

\section{Methods}

\section{Participants}

As a priori calculation of required sample size for the twoway interaction resulted in a minimum of $\mathrm{N}=53$ for a power of $1-\beta=0.95$, and an effect of $d=0.5$ - an effect size which can be expected in uncontrollable, socialevaluative tasks like TSST. ${ }^{4}$ A total of 60 university students between the ages of 18 and 30 years old $(50 \%$ female) participated in the experiment. They were randomly divided into two groups: TSST-VR (stress group) or placebo (non-stress group). Three participants were excluded because they did not finish the subjective scales, or had a lot of artifacts in the recorded electrocardiogram (ECG) signal. The final effective data came from 57 participants. The stress group contained 15 males and 14 females, with a mean age of 23.5 years $(S D=2.35)$. The non-stress group included 13 males and 15 females, with a mean age of 23.04 years $(\mathrm{SD}=1.88)$.

The education level of participants was bachelor's degree or above. They were all right-handed. To be eligible to participate, participants needed to meet the following criteria: no cardiovascular and endocrine disorders, no psychiatric or family medical history, normal or correctedto-normal vision and hearing. The females included in the present study reported not using mood-altering medication (such as amphetamines, coffee and alcohol) or oral contraceptives, and having had a normal menstrual cycle for the previous four months. The study was scheduled according to the diurnal cortisol curve, between 2:00 pm and 4:00 pm, when levels of salivary cortisol are more stable. In addition, none of the females were menstruating at the time of the study, with 15 being in the luteal phase and 15 in the follicular phase of their menstrual cycles. On arrival at the laboratory, all participants provided written informed consent to participate in the experiment, and received monetary compensation $(\$ 15)$ after the experiment for participating. The study was in conformance with the guidelines of the Declaration of Helsinki. Experimental procedures were approved by the Institutional Review Board of the State Key Laboratory of Cognitive Neuroscience and Learning of Beijing Normal University.

\section{Materials}

\section{Subjective Scales}

Visual Analog Scale for Stressful Situations (VAS)

A VAS is a $100 \mathrm{~mm}$ bipolar line that measures a characteristic across a continuum. ${ }^{16}$ One end of the line is zero, indicating no stress; and the other end of the line is $10 \mathrm{~cm}$, indicating unbearable stress. The participants marked a spot on the line resembling their subjective appraisal of stress perception.

\section{Emotional Scales}

The emotional scales included the Beck Anxiety Inventory (BAI), ${ }^{37}$ the Beck Depression Inventory (BDI), ${ }^{38}$ and the Positive Affect and Negative Affect Scale (PANAS). ${ }^{39}$ The BAI comprises 21 items. It utilizes a four-level scoring rubric in which " 1 " indicates no discomfort. The BDI also comprises 21 items and for each item, respondents need to endorse one of four possible responses. The PANAS includes 20 items and contains two emotion dimensions (positive, 10 items; negative, 10 items). The PANAS is a Likert-style questionnaire from 1 (not at all) to 5 (extremely).

\section{Simplified Coping Stress Questionnaire (SCSQ)}

The SCSQ comprises 20 items including 12 positive and eight negative items, and yielding two coping dimensions. The SCSQ is also a Likert-style questionnaire ranging from 1 (no use) to 4 (always use). ${ }^{40}$

\section{The Trier Social Stress Test in Virtual Reality Environment (TSST-VR)}

The WorldViz Vizard 4.0 software, the PPT E8 optical inertial hybrid wide-area tracking system and the nVisor SX60 head-mounted display were used to present the virtual audience. ${ }^{10}$ The virtual room had a table, four 
chairs (one real chair for the participant), a surveillance camera and a microphone (height-adjustable). The committee comprised three virtual persons with neutral facial expressions (a middle-aged man in the middle, a young woman to the left and a young man to the right, all sitting down behind a table, facing the participant). The virtual persons nod, look at the participant or elsewhere and move their feet during the test to improve the realism of the VR simulation (Figure 1).

\section{Procedure}

\section{The Initial Assessment}

Once the participants arrived at the laboratory (Room A), they were given a consent inform to be signed and brief information about the experiment. Afterwards, the initial assessment was conducted on them. They completed the following scales: BAI, BDI, PANAS, SCSQ and VAS. Then, the participants were attached to the equipment of physiological recording (five-minutes resting state data; baseline). The physiological indices included EDA, HR and HRV. Then, the participants were led to Room B to participate in the virtual reality test (stress or control conditions).

\section{Virtual Reality Period}

The participants were randomly assigned to stress (TSST) and non-stress (placebo) group during the virtual reality period. The experimenter took the participants to the virtual reality room (Room B), gave the helmet to the participants and explained the introductions. The specific procedures were as follows:

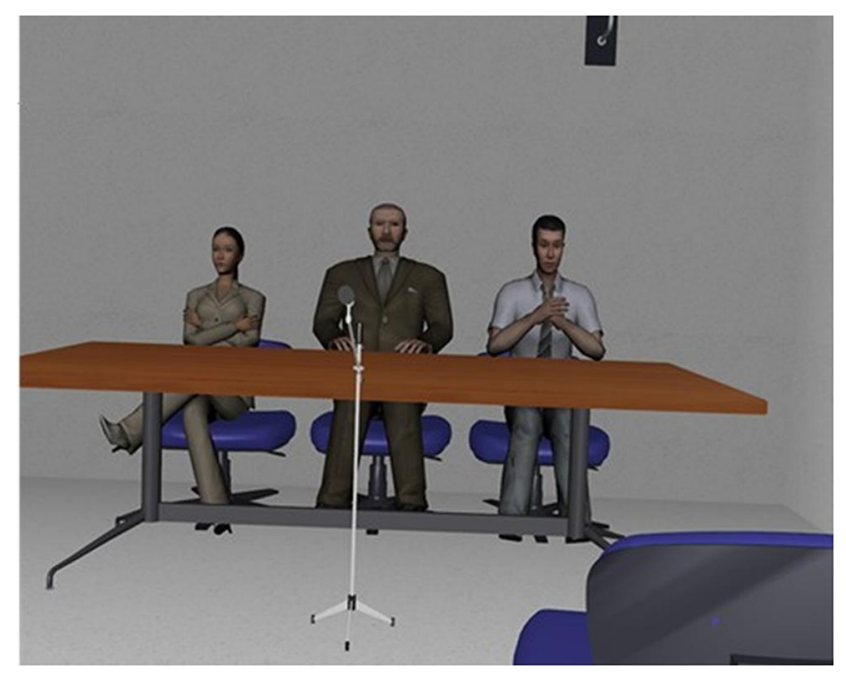

Figure I The figure shows the committee in front of a participant.

\section{Stress Group (TSST)}

The instructions were as follows: You should give a public speech for running for monitor in your class, in front of the committee for five minutes, to showcase the positive traits of your character that could give you an advantage over other candidates. After that, the participants were also told that after the speech, the committee would give them another task.

\section{Preparation for the Speech}

Preparation for the speech included voices coming from within the helmet, by virtual persons, requesting the participants to prepare a speech about running for the position. The voices were controlled by the experimenter with a remote keyboard which the participants could not see. A surround-sound system played the recorded voices in advance. When the participants wore the helmet and sat in the virtual room, they would hear the young man on the right, among the three committee members, say "You have three minutes to prepare for the speech." After the three minutes, the man would say "Time is up, please step up to the microphone and begin the speech in a clear, loud voice."

\section{Public Speech}

Mostly, the participants finished talking after within two to three minutes after beginning the speech. After that, the older man in the middle place of the committee members would say "There is still time left" or "Please go on, when the time is up, I will tell you". When the participants would halt for a second time before the first five minutes were over, all the committee members would look at the participants with a neutral facial expression, without talking, for 20 seconds. If the participants experienced difficulties or struggled with the speech, the young woman on the left would ask them "Do you have a similar campaign experience?" or "If you don't apply for the position, so what?." If the participants asked a question to the committee members, they would respond "I'm sorry, I can't answer that question."

\section{Mental Arithmetic}

After the public speech, the man in the middle would say "OK, thank you for your speech. Let's conduct the next task". The participants in this group would have to serially subtract 13, starting at 2011, for five minutes. They were told that upon each error, a committee member would ask them to start anew, from the beginning. After the mental arithmetic task, the man in the middle would say "OK, this 
is it for today's interview. You can go now." Then, the experimenter would approach the participants, take off their helmets, and guide them back into Room A.

\section{Non-Stress Group (Placebo)}

The virtual room was the same as the one used for the stress group, except that it was empty, and there was only one chair (in the same position as the chair in the TSST condition).

The instructions were as follows: "You should talk freely about yourself for five minutes, and then complete a simple math task for five minutes."

\section{Free Talking}

The participants sat on a chair for three minutes to prepare. Following that, they would hear a voice coming from within their helmet that would say Stand up. You can choose any location in the room. Stand up and speak aloud for five minutes. The content of the speech can be a movie you have seen, or a novel you have read recently, or a recent vacation trip.

\section{Math Task}

After five minutes, the experimenter would say to the participants OK, now we have another task. You have to keep standing. Starting with zero, add 15 continuously. The task will last for five minutes. After this, you have to report your results to me. OK, let's start. Five minutes later, the experimenter would walk to the participants, signaled them to help him take off the helmet, asked for the result of the math task and then lead the participants back to Room A.

\section{The Final Assessment}

The participants filled in the VAS again. They were then recorded again for five minutes of physiological activity, for the recovery reactivity. A diagram of the protocol of TSST-VR is shown in Figure 2.

\section{Data Recording}

Participants wore an nVisor SX60 head-mounted display (HMD, NVIS, Reston, VA) that featured dual 1280 horizontal by 1024 vertical pixel resolution panels that refreshed at $60 \mathrm{~Hz}$ (WorldViz, Santa Barbara, CA, USA). Stereoscopic images were rendered by a $2.8 \mathrm{G}$ CPU Intel computer with a $4 \mathrm{G}$ graphics card.

Physiological activity data were collected via the BioNomadix remote tracking system (BIOPAC MP150, Biopac Systems, Inc., Goleta, CA). The bipolar electrodes that were used to collect the wireless electrodermal activity (EDA100C) were attached to the ring and middle fingers of the subjects' left hands (VIN+ and VIN-, respectively). The sample rate was $250 \mathrm{~Hz}$, and the units were in $\mu \mathrm{mho} / \mathrm{V}$.

ECG signal data are transmitted at a rate of $2000 \mathrm{~Hz}$. The heart rate (HR) of each participant was obtained based on the R-R interval, which was immediately extracted from the ECG signal. We used the Acknowledge 4.2 software to extract and analyze the EDA, HR and HRV. The Fast Fourier Transformation (FFT) was used to transform the R-R interval, which converted from the raw $\mathrm{R}$ wave to the HRV frequency domain information. The unit of the

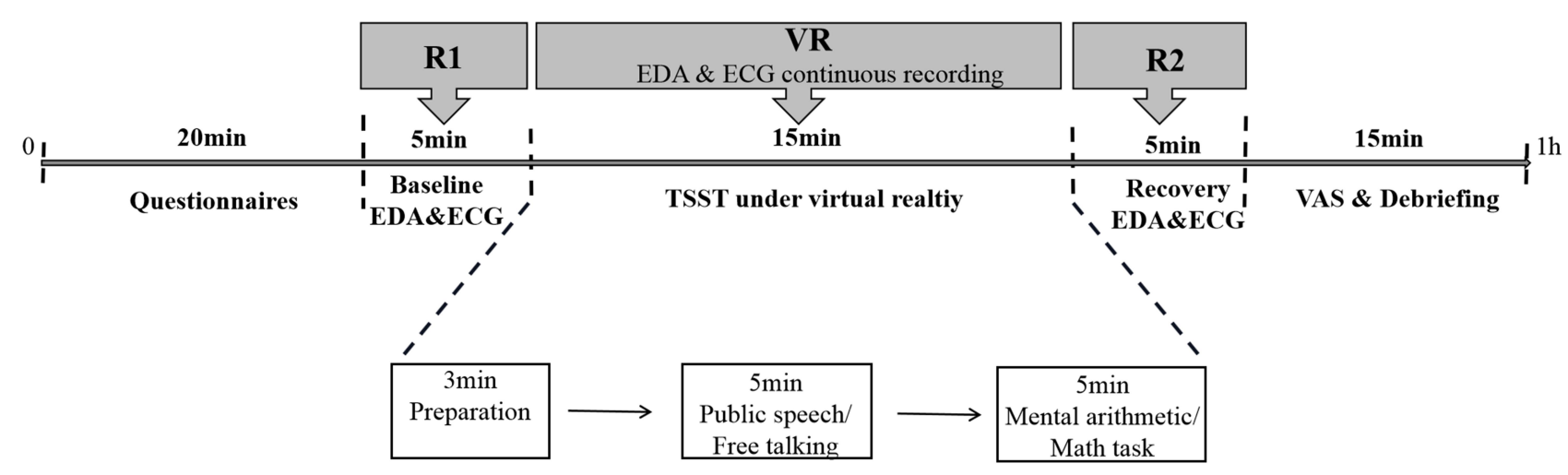

Figure 2 The protocol of a virtual reality of the Trier Social Stress Test (TSST-VR).

Notes: After arrival, participants filled in questionnaires (BAI, BDI, PANAS, SCSQ and VAS) for about 20 minutes and then they were attached to the equipment of physiological recording for about 25 minutes. During this 25 -minutes, they were recorded the first five-minutes resting state data for baseline EDA and ECG (the time point RI). Then they were continually recorded when conducting the VR protocol (the time period VR) and after VR protocol, the participants were recorded for another fiveminutes resting state data for recovery EDA and ECG (the time point R2). At last, the participants filled in VAS again and were given debriefing of the experiment (about 20 minutes). The whole experiment lasted for about one hour.

Abbreviations: BAI, Beck Anxiety Inventory; BDI, Beck Depression Inventory; PANAS, Positive Affect and Negative Affect Scale; SCSQ, Simplified Coping Style Questionnaire; VAS, Visual Analog of Stress; EDA, electrodermal activity; ECG, electrocardiogram; TSST, Trier Social Stress Test; Placebo, the control condition of the TSST, which comprised free talking (5 minutes) and simple math (5 minutes). 
HR was beats per minute (bpm). The electrode connection followed the precordial lead way.

\section{Data Analysis}

The SPSS16.0 software (SPSS Inc., Chicago, IL) was used to process and analyze data. We conducted a mixed-factor ANOVA on the sex differences in stress reactivity to the TSST-VR for which the test time (baseline, VR and recovery phases) was the within-subjects variable. The distributions were tested for normality and homoscedasticity purposes before the ANOVAs were conducted. All of the significant analyses used the two-way test $(\mathrm{p}<0.05)$, the partial eta squared $\left(\eta^{2}\right)$ and Cohen's $d$ for the effect size. We adopted the logarithmic transformation to normalize the HRV data because of the skewness of data distribution. $^{41}$ The data were all presented as the mean $\pm \mathrm{SD}$.

\section{Results}

\section{Sex Differences on Coping and Emotional States}

The independent sample $t$-tests were conducted on the scores of the SCSQ, BAI, BDI and PANAS for men and women. The results showed that men and women had no significant differences on the scores of the BAI, BDI, PANAS and SCSQ (Table 1).

\section{Stress Reactivity to TSST-VR}

\section{The Subjective Stress Reactivity to TSST-VR}

To evaluate the effect of the TSST-VR on stress perception, a mixed-factor ANOVA was conducted on VAS measures, with TIME (before and after the VR) as within-subject variable, and GROUP (stress, non-stress) and SEX (male, female) as between-subjects variables.

Table I The Scores of Males $(\mathrm{N}=28)$ and Females $(\mathrm{N}=29)$ of Emotion Scales (BAI, BDI, PANAS) and Coping Scale (SCSQ)

\begin{tabular}{|c|c|c|c|}
\hline Scales & Male (M $\pm S D)$ & Female (M士SD) & $p$ \\
\hline BAI & $32.6 I \pm 7.4 I$ & $30.59 \pm 5.32$ & 0.394 \\
\hline $\mathrm{BDI}$ & $8.79 \pm 7.50$ & $6.48 \pm 5.60$ & 0.320 \\
\hline Positive Affect & $29.18 \pm 9.62$ & $28.17 \pm 7.98$ & 0.638 \\
\hline Negative Affect & $17.82 \pm 5.38$ & $16.83 \pm 4.53$ & 0.585 \\
\hline Positive Coping & $21.21 \pm 6.02$ & $20.34 \pm 5.86$ & 0.394 \\
\hline Negative Coping & $9.00 \pm 3.91$ & $9.10 \pm 3.63$ & 0.907 \\
\hline
\end{tabular}

Notes: Values are mean \pm S.D. The BAI is the Beck Anxiety Inventory and the BDI is the Beck Depression Inventory; Positive Affect and Negative Affect are subscales of PANAS, Positive Coping and Negative Coping are subscales of SCSQ.
The results showed that the main effects of group $\left(\mathrm{F}_{(1,53)}=\right.$ 14.53, $p<0.001, \eta 2=0.22)$ and $\operatorname{sex}\left(\mathrm{F}_{(1,53)}=4.11, p=0.048, \eta^{2}=\right.$ $0.07)$ were significant whereas the time main effect on the VAS was not significant, $\mathrm{F}_{(1,53)}=3.25, p=0.077, \eta^{2}=0.06$. The only significant interaction that we obtained was the TIME $\times$ GROUP on the VAS, $\mathrm{F}_{(1,53)}=6.57, p=0.013, \eta^{2}=$ 0.11 . Further simple effect analysis revealed that compared to the non-stress group $(\mathrm{M}=37.25 \mathrm{~mm}, \mathrm{SD}=14.43 \mathrm{~mm})$, the stress group $(\mathrm{M}=56.75 \mathrm{~mm}, \mathrm{SD}=17.55 \mathrm{~mm})$ reported higher scores on the VAS after the VR, $\mathrm{F}_{(1,55)}=10.16, p=0.002$ (Figure 3).

\section{The Objective Stress Reactivity to TSST-VR}

A mixed-factor ANOVAs was carried out, with TIME (baseline, R1; virtual reality, VR; recovery, R2) as a withinsubject variable, and GROUP (stress, non-stress) and SEX (male, female) as between-subjects variables, to test sex differences on objective stress reactivity (EDA, HR and HRV) to TSST-VR. The analysis revealed significant main effects of TIME on EDA $\left(\mathrm{F}_{(2,106)}=31.30, p<0.001, \eta^{2}=\right.$ $0.371), \operatorname{HR}\left(\mathrm{F}_{(2,106)}=42.98, p<0.001, \eta^{2}=0.45\right)$ and HRV $\left(\mathrm{LF}: \mathrm{F}_{(2,106)}=10.46, p<0.001, \eta^{2}=0.17\right.$; HF: $\mathrm{F}_{(2,106)}=$ 9.43, $\left.p<0.001, \eta^{2}=0.15\right)$. In addition, the main effects of $\operatorname{SEX}$ on $\operatorname{EDA}\left(\mathrm{F}_{(1,53)}=4.45, p=0.040, \eta^{2}=0.08\right)$, HR $\left(\mathrm{F}_{(1,53)}=4.77, p=0.033, \eta^{2}=0.08\right)$, and the LF/HF of HRV $\left(\mathrm{F}_{(1,53)}=10.36, p=0.002, \eta^{2}=0.16\right)$ were also significant.

The further analysis of TIME main effect on HRV showed that (paired $t$-test), there were significant differences on HRV $\left(\mathrm{LF}, \mathrm{t}_{(56)}=-3.53, p=0.001 ; \mathrm{HF}, \mathrm{t}_{(56)}=\right.$ $-3.11, p=0.003$ ) between $\mathrm{R} 1$ and $\mathrm{R} 2$ time points. There were also significant differences on $\operatorname{HRV}\left(\mathrm{LF}, \mathrm{t}_{(56)}=-4.13\right.$,

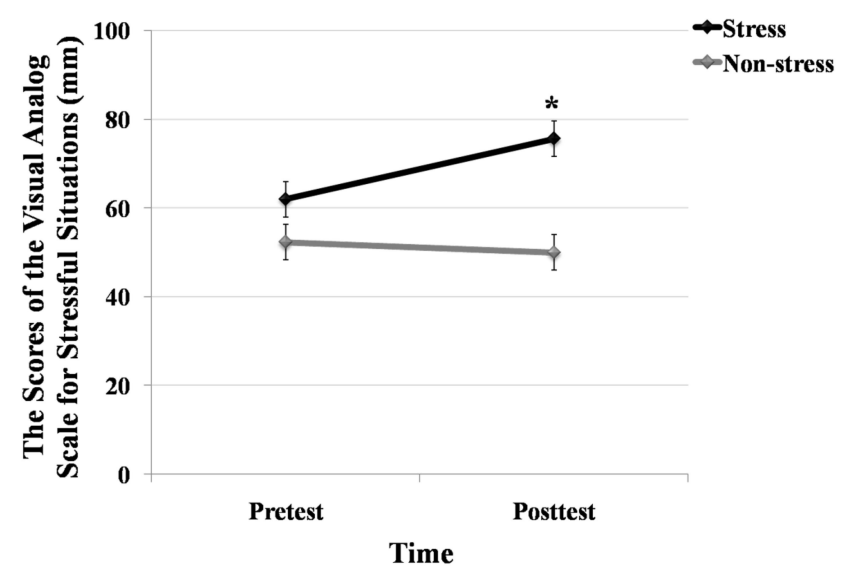

Figure 3 The scores on the VAS (visual analog scale for stressful situations) of men and women from the stress $(\mathrm{N}=28)$ or non-stress $(\mathrm{N}=29)$ groups before and after the virtual reality environment (VR).

Note: Statistical level, $* \mathrm{p}<0.05$ 
Table 2 The Low Frequency (LF), High Frequency (HF) of Heart Rate Variability (HRV) and the Ration of LF and HF (LF/HF) in Different Time Points (RI, VR and R2)

\begin{tabular}{|l|l|l|l|}
\hline Index & RI & $\begin{array}{l}\text { Time } \\
\text { VR }\end{array}$ & R2 \\
\hline LF (nu) & $-3.63 \pm 0.09$ & $-3.22 \pm 0.09$ & $-3.76 \pm 0.08$ \\
HF (nu) & $-3.70 \pm 0.10$ & $-3.29 \pm 0.10$ & $-3.83 \pm 0.09$ \\
LF/HF & $1.65 \pm 2.01$ & $1.55 \pm 1.92$ & $1.70 \pm 1.63$ \\
\hline
\end{tabular}

Abbreviations: LF, low frequency of HRV; HF, high frequency of HRV; LF/HF, the ration of LF and HF; VR, virtual reality; RI, the first resting state (before VR conduction, five minutes); R2, the second resting state (after VR conduction, five minutes).

$\left.p<0.001 ; \mathrm{HF}, \mathrm{t}_{(56)}=-4.11, p<0.001\right)$ between $\mathrm{VR}$ and $\mathrm{R} 2$ time points (Table 2).

The further analysis of SEX main effects on EDA, HR and the LF/HF of HRV showed that (independent $t$-test), in the $\mathrm{R} 1$ time point, the $\operatorname{EDA}\left(\mathrm{t}_{(55)}=2.14, p=0.037\right)$ and $\mathrm{LF} / \mathrm{HF}$ of $\mathrm{HRV}\left(\mathrm{t}_{(55)}=2.10, p=0.04\right)$ were different for male and female participants. In the VR time point, the HR was different for male and female participants $\left(\mathrm{t}_{(55)}=\right.$ $-2.44, p=0.018)$. At last, in the $\mathrm{R} 2$ time point, the HR $\left(\mathrm{t}_{(55)}=-2.39, p=0.020\right)$ and LF/HF of HRV $\left(\mathrm{t}_{(55)}=3.12\right.$, $p=0.003$ ) were different for male and female participants (Figure 4).

\section{Effects of Emotional States and Coping on Stress Reactivity to TSST-VR}

The Pearson's correlations were conducted on the data of subjective scales and the physiological activity at different time points (R1, VR and R2). The results of correlations showed that firstly, in R1 and VR time points, the EDA and anxiety (BAI) had a positive correlation. Secondly, in the VR, the HRVs (LF and HF) were negatively correlated to depression (BDI) and negative affect (NA). Finally, in $\mathrm{R} 2$, HR and depression exhibited a negative correlation;

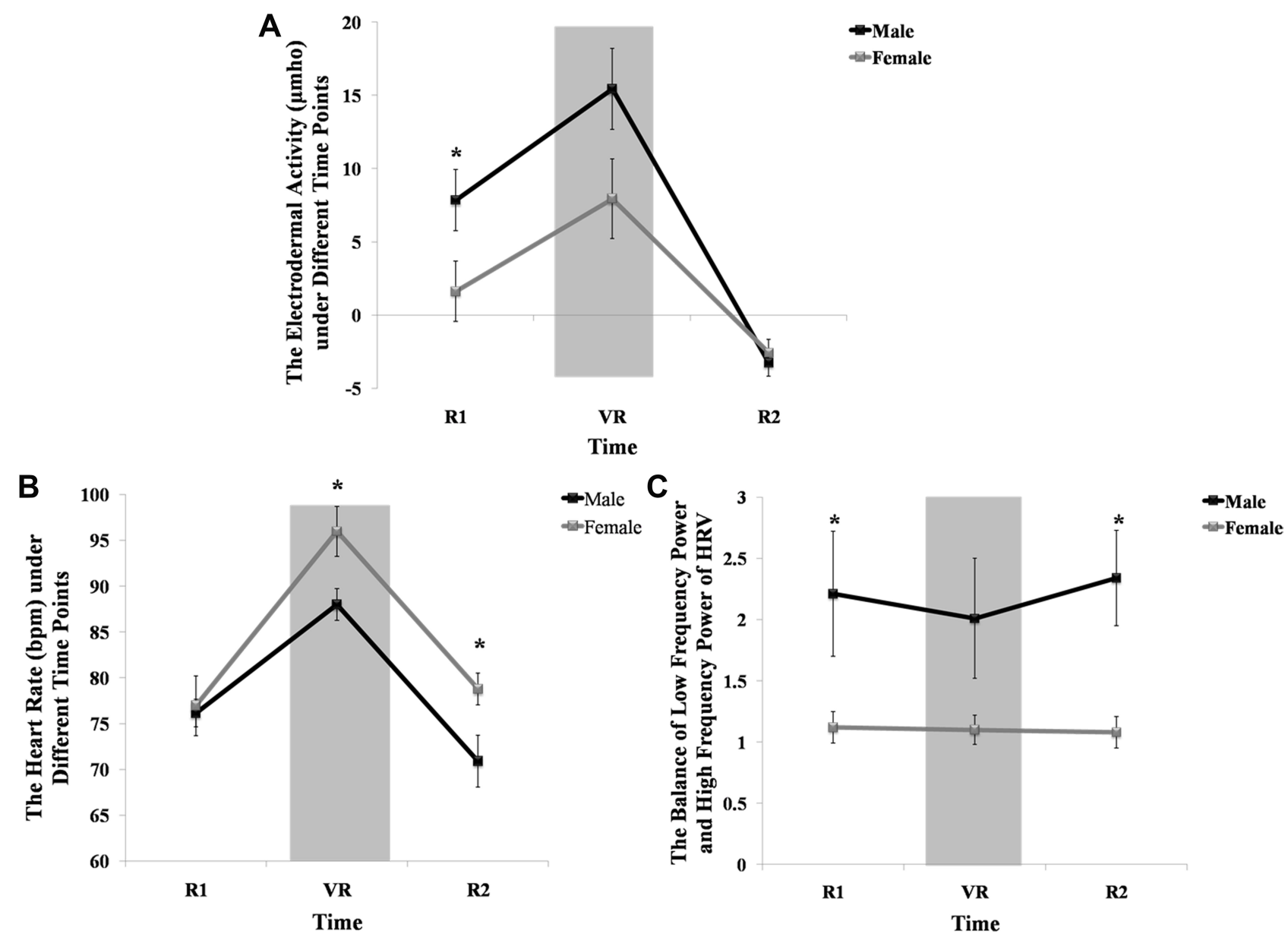

Figure 4 The electrical activity (EDA, $\mu \mathrm{hmo})$, heart rate (HR, bpm) and the ratio of low power and high power of heart rate variability (balance of HRV) for men and women in the stress $(\mathrm{N}=28)$ or non-stress $(\mathrm{N}=29)$ groups during different test time phases (baseline, virtual reality and recovery). (A) The EDA ( $\mu$ hmo) of men and women from the stress and non-stress groups in different test times. (B) The HR (bpm) of men and women in the stress and non-stress groups during different test times. (C) The balance of HRV for men and women in the stress and non-stress groups during different test times.

Note: Statistical level, ${ }^{*} \mathrm{p}<0.05$ 
Table 3 The Pearson's Correlations Between Subjective Scales (BAI, BDI, EPQ, PANAS and SCSQ) and Physiological Activities (EDA, HR, LF, HF) During the First Resting State (RI), The Second Resting State (R2) and Virtual Reality (VR) (N=57)

\begin{tabular}{|l|l|l|l|l|l|}
\hline Time & Index & BAI & BDI & NA & PC \\
\hline RI & EDA & $0.357(0.006)^{* *}$ & & & \\
\hline R2 & HR & & $-0.256(0.054)$ & $-0.349(0.008)^{* *}$ \\
& LF & $0.277(0.037)^{*}$ & $-0.352(0.007)^{* *}$ \\
\hline VR & HF & & & \\
& EDA & & $-0.384(0.003)^{* *}$ & $-0.305(0.021)^{*}$ & \\
& LF & & $-0.326(0.013)^{*}$ & & \\
\hline
\end{tabular}

Note: Statistical level, ${ }^{*} p<0.05,{ }^{*} p<0.01$

the HRVs (LF) were positively correlated to depression and negatively correlated to positive coping (PC). The specific trends are presented in Table 3 .

\section{Discussion}

The present study assessed whether a modified version of TSST, which uses a placebo as control condition and conducts in virtual, induces psychosocial stress on men and women in the laboratory. The results demonstrated that, compared to the non-stress group, the stress group felt more stress after VR. Compared to women, men had stronger EDA before VR; lower HR under VR; and higher HRV after VR. The EDA before and under VR were negatively correlated with anxiety. The HRV under VR had negative correlations with depression and negative affect. After VR, the HRV was positively correlated with depression and negatively correlated with the positive coping style.

In this study, we utilized an experimental condition which steadily induced participants' stress reactivity to the TSST-VR. This is consistent with the studies of Kelly et al, Kotlyar et al and Jonsson et al, which used the virtual reality environment to conduct the $\operatorname{TSST}^{7-9}$ In the present study, the comparison between the TSST-VR and the control condition in VR, showed differential reaction patterns. The fact that we found stress effects on our dependent measures only in the stress condition, suggests that the immersion in a foreign virtual environment is not sufficient to elicit a stress response, at least in terms of stress perception. Meanwhile, the use of a placebo as control condition gave us the advantage of reducing procedure variability, while maintaining the social-evaluative and uncontrollable nature of the task. ${ }^{15}$ Taken together, we demonstrated that, while participants showed an increase of stress levels for almost all our psychobiological stress markers, no such rise was observed in our control groups. To the best of our knowledge, this is the first study to date to have used a placebo as control condition in a TSST-VR paradigm.

We used the wireless polygraph system to record participants' physiological changes throughout the experiment, consistent with Hellhammer and Schubert, ${ }^{16}$ who posited that the dynamic measurement of stress reactivity (before, during and after the TSST) would provide the most details. The correlation between subjective and objective reactivity to the TSST-VR in our study, connected sympathetic activation with diverse psychological variables regarding anxiety, depression, negative affect and coping styles. Specifically, we observed that EDA, before and during the VR, was positively correlated with participants' reported anxiety, suggesting that those who demonstrated more sympathetic activation, before and during VR, were more likely to experience anxiety. These results were in line with those of Ruiz et $\mathrm{al}^{12}$ and Montero-López et al. ${ }^{31}$ The negative correlation of HRV under VR, and the negative affect in our study duplicates the results of Child et al, ${ }^{42}$ who observed that the TSST induced participants' cardiovascular reactivity and increased their negative affect. Moreover, after VR, the HRV was positively correlated with depression and negatively correlated with the positive coping style. These results are consistent with the trajectory of HRV across different timepoints of $\operatorname{TSST}^{35}$ and further indicate that examining HRV in response to stress contributes to independent information.

Focusing on EDA, HR and HRV as major physiological stress markers, the results are indeed consistent with the assumption that, a reliable stimulation of the cardiovascular reactivity with a social-evaluative stressor, is possible in VR. ${ }^{43}$ Specifically, compared to women, men 
had stronger EDA before VR, lower HR under VR, and higher HRV after VR. These results contribute to a greater understanding of sex-specific physiologic reactivity to psychosocial stressors. However, Santl et $a 1,{ }^{32}$ compared stress responses among 16 women taking oral contraceptives and 16 men in TSST-VR, and demonstrated that the physiological measures (HR and skin conductance level) did not discriminate between men and women. While the present study only recruited female participants who were not using any oral contraceptives or menstruating at the time the TSST-VR was conducted, these inconsistent inclusion criteria may uncover important modulation of stress metrics, such as HRV, by gonadal hormones.

Despite its strengths, the current study has limitations that need to be taken into account. Since we did not directly compare the physiological response to TSST-VR to TSST in vivo, we cannot determine which provokes a greater physiological response. And we did not strictly control for state covariates (eg, prior day experiences, sleep hygiene), the possibility of state-related confounding may occur when explaining the physiological variation we observed. ${ }^{14,44}$ Additionally, although with the intention of deducing sex-specific indices and choosing female participants who were not menstruating or taking oral contraceptives, we observed sex differences of sympathetic activation in TSST-VR. Future studies should further investigate the effects of menstrual cycle phases on stress responses in TSST-VR, which could give an insight into the intra- and inter-individual variations in the psychobiological stress responses elicited.

Overall, in the present study, we presented an adaptation of TSST which used placebo as control condition in virtual reality to investigate the effects of psychosocial stressor on response pattern for men and women. The analysis of different physiological and subjective measures revealed different aspects of the exposure to a psychosocially stressful situation. Stress perception could be observed with our TSST-VR induction which indicated that virtual reality simply provides enough visual information to stimulate the schemas of a social-evaluative threat. Meanwhile, the dissociation of self-reported and physiological stress reactions between men and women warrants further study.

\section{Data Sharing Statement}

All data, models, and code generated or used during the study appear in the submitted article.

\section{Ethics Approval and Consent to Participate}

All participants provided written informed consent to participate the present experiment. The study conformed to the provisions of the Declaration of Helsinki. Experimental procedures were approved by the Institutional Review Board of the State Key Laboratory of Cognitive Neuroscience and Learning of Beijing Normal University (ICBIR_B_0010_005).

\section{Consent for Publication}

Not applicable.

\section{Acknowledgments}

The authors thank Prof. Tian Po Oei for his help in the modification of the paper, which significantly enhanced of this manuscript. The authors would like to express their gratitude for his work.

\section{Author Contributions}

All authors made a significant contribution to the work reported, whether that is in the conception, study design, execution, acquisition of data, analysis and interpretation, or in all these areas; took part in drafting, revising or critically reviewing the article; gave final approval of the version to be published; have agreed on the journal to which the article has been submitted; and agree to be accountable for all aspects of the work.

\section{Funding}

This research did not receive any specific grant from funding agencies in the public, commercial, or not-forprofit sectors.

\section{Disclosure}

The authors report no conflicts of interest for this work.

\section{References}

1. McEwen BS. Protective and damaging effects of stress mediators: central role of the brain. Dialogues Clin Neurosci. 2006;8:367-381.

2. Armario A, Labad J, Nadal R. Focusing attention on biological markers of acute stressor intensity: empirical evidence and limitations. Neurosci Biobehav Rev. 2020;111:95-103. doi:10.1016/j.neubiorev. 2020.01 .013

3. Goodman WK, Janson J, Wolf JM. Meta-analytical assessment of the effects of protocol variations on cortisol responses to the Trier Social Stress Test. Psychoneuroendocrinology. 2017;80:26-35. doi:10.1016/j. psyneuen.2017.02.030 
4. Dickerson SS, Kemeny ME. Acute stressors and cortisol responses: a theoretical integration and synthesis of laboratory research. Psychol Bull. 2004;130:355-391. doi:10.1037/0033-2909.130.3.355

5. Zorn JV, Schür RR, Boks MP, Kahn RS, Joëls M, Vinkers CH. Cortisol stress reactivity across psychiatric disorders: a systematic review and meta-analysis. Psychoneuroendocrinology. 2017;77:25-36. doi:10.10 16/j.psyneuen.2016.11.036

6. Kischbaum C, Pirke K-M, Hellhammer DH. The 'trier social stress test' - a tool for investigating psychobiological stress responses in a laboratory setting. Neuropsychobiology. 1993;28:76-81. doi:10.1159/000119004

7. Kelly O, Matheson K, Martinez A, Merali Z, Anisman H. Psychosocial stress evoked by a virtual audience: relation to neuroendocrine activity. Cyber Psychology Behavior. 2007;10:655-662. doi:10.1089/cpb.2007.9973

8. Kotlyar M, Donahue C, Thuras P, et al. Physiological response to a speech stressor presented in a virtual reality environment. Psychophysiology. 2008;45:1034-1037. doi:10.1111/j.14698986.2008.00690.x

9. Jonsson P, Wallergard M, Osterberg K, Hansen AM, Johansson G, Karlson B. Cardiovascular and cortisol reactivity and habituation to a virtual reality version of the Trier Social Stress Test: A pilot study. Psychoneuroendocrinology. 2010;35:1397-1403. doi:10.1016/j. psyneuen.2010.04.003

10. Wallergård M, Jönsson P, Johansson G, Karlson B. A virtual reality version of the Trier Social Stress Test: a pilot study. Presence Teleop Virt. 2011;20(4):325-336. doi:10.1162/PRES_a_00052

11. Shiban Y, Diemer J, Brandl S, Zack R, Mühlberger A, Wüst S. Trier social stress test in vivo and in virtual reality: dissociation of response domains. Int $J$ Psychophysiol. 2016;110:47-55. doi:10.1016/j.ijpsycho.2016.10.008

12. Ruiz AS, Peralta-Ramirez MI, Garcia-Rios MC, Munoz MA, Navarrete-Navarrete N, Blazquez-Ortiz A. Adaptation of the trier social stress test to virtual reality: psycho-physiological and neuroendocrine modulation. $J$ CyberTherapy Rehabilitation. 2010;3:405-415.

13. Zimmer P, Buttlar B, Halbeisen G, Walther E, Domes G. Virtually stresses? A refined virtual reality adaptation of the Trier Social Stress Test (TSST) induces robust endocrine responses. Psychoneuroendocrinology. 2019;101:186-192. doi:10.1016/j. psyneuen.2018.11.010

14. Narvaez Linares NF, Charron V, Ouimet AJ, Labelle PR, Plamondon H. A systematic review of the Trier Social Stress Test methodology: issues in promoting study comparison and replicable research. Neurobiol Stress. 2020;13:100235. doi:10.1016/j. ynstr.2020.100235

15. Het S, Rohleder N, Schoofs D, Kischbaum C, Wolf OT. Neuroendocrine and psychometric evaluation of a placebo version of the 'trier social stress test'. Psychoneuroendocrinology. 2009;34:1075-1086. doi:10.1016/j.psyneuen.2009.02.008

16. Hellhammer J, Schubert M. The physiological response to Trier Social Stress Test relates to subjective measures of stress during but not before or after the test. Psychoneuroendocrinology. 2012;37:119-124. doi:10.1016/j.psyneuen.2011.05.012

17. Allen AP, Kennedy PJ, Cryan JF, Dinan TG, Clarke G. Biological and psychological markers of stress in humans: focus on the Trier Social Stress Test. Neurosci Biobehav Rev. 2014;38:94-124. doi:10.1016/j.neubiorev.2013.11.005

18. Kim HG, Cheon EJ, Bai DS, Lee YH, Koo BH. Stress and heart rate variability: a meta-analysis and review of the literature. Psychiatry Investig. 2018;15(3):235-245. doi:10.30773/pi.2017.08.17

19. Thayer JF, Ash F, Fredrikson M, Sollers JJ, Wager TD. A meta-analysis of heart rate variability and neuroimaging studies: implications for heart rate variability as a marker of stress and health. Neurosci Biobehav Rev. 2012;36(2):747-756. doi:10.1016/j. neubiorev.2011.11.009
20. Xhyheri B, Mandrini O, Mazzolini M, Pizzi C, Bugiardini R. Heart rate variability today. Prog Cardiovasc Dis. 2012;55:321-331. doi:10.1016/j.pcad.2012.09.001

21. Mostajeran F, Balci MB, Sterinicke F, Kuhn S, Gallinat J The effects of virtual audience size on social anxiety during public speaking. 2020 IEEE Conference on Virtual Reality and 3D User Interfaces (VR), Atlanta, GA, USA. 2020; 303-312.

22. Yim IS, Quas JA, Cahill L, Hayakawa CM. Children's and adults' salivary cortisol responses to an identical psychosocial laboratory stressor. Psychoneuroendocrinology. 2010;35:241-248. doi:10.1016/ j.psyneuen.2009.06.014

23. Allen AP, Kennedy PJ, Dockray S, Cryan JF, Dinan TG, Clarke G. The trier social stress test: principles and practice. Neurobiol Stress. 2017;6:113-126. doi:10.1016/j.ynstr.2016.11.001

24. Labuschagne I, Grace C, Rendell P, Terrett G, Heinrichs M. An introductory guide to conducting the TrierSocial Stress Test. Neurosci Biobehav Rev. 2019;107:686-695. doi:10.1016/j. neubiorev.2019.09.032

25. Kudielka BM, Hellhammer D, Wüst S. Why do we respond so differently? Reviewing determinants of human salivary cortisol responses to challenge. Psychoneuroendocrinology. 2009;34:2-18. doi:10.1016/j.psyneuen.2008.10.004

26. Liu JJW, Ein N, Peck K, Huang V, Pruessner JC, Vickers K. Sex differences in salivary cortisol reactivity to the Trier Social Stress Test (TSST): a meta-analysis. Psychoneuroendocrinology. 2017;82:26-37. doi:10.1016/j.psyneuen.2017.04.007

27. Strahler J, Skoluda N, Kappert MB, Nater UM. Simultaneous measurement of salivary cortisol and alpha-amylase: application and recommendations. Neurosci Biobehav Rev. 2017;83:657-677. doi:10.1016/j.neubiorev.2017.08.015

28. Schmalenberger KM, Eisenlohr-Moul TA, Wurth L, et al. A systematic review and meta-analysis of within-person changes in cardiac vagal activity across the menstrual cycle: implications for female health and future studies. J Clin Med. 2019;8:1946. doi: $10.3390 /$ jcm8111946

29. Zänkert S, Bellingrath S, Wüst S, Kudielka BM. HPA axis responses to psychological challenge linking stress and disease: what do we know on sources of intra- and interindividual variability? Psychoneuroendocrinology. 2019;105:86-93.

30. Helminen EC, Morton ML, Wang Q, Felver JC. A meta-analysis of cortisol reactivity to the Trier Social StressTest in virtual environments. Psychoneuroendocrinology. 2019;110(104437).

31. Montero-López E, Santos-Ruiz A, García-Ríos M, RodríguezBlázquez R, Pérez-García M, Peralta-Ramírez M. A virtual reality approach to the Trier Social Stress Test: contrasting two distinct protocols. Behav Res. 2016;48(1):223-232. doi:10.3758/s13428015-0565-4

32. Santl J, Shiban Y, Plab A, Wust S, Kudielka BM, Muhlbergr A. Gender differences in stress responses during a virtual reality trier social stress test. Int J Virtual Real. 2019;19(2):2-15.

33. Montero-López E, Santos-Ruiz A, García-Ríos MC, RodríguezBlázquez M, Rogers HL, Peralta-Ramírez MI. The relationship between the menstrual cycle and cortisol secretion: daily and stress-invoked cortisol patterns. Int $J$ Psychophysiol. 2018;131:67-72. doi:10.1016/j.ijpsycho.2018.03.021

34. Bale TL, Epperson CN. Sex differences and stress across the lifespan. Nat Neurosci. 2015;18(10):1413-1420. doi:10.1038/nn.4112

35. Hamidovic A, Van Hedger K, Choi SH, Flowers S, Wardle M, Childs E. Quantitative meta-analysis of heart rate variability finds reduced parasympathetic cardiac tone in women compared to men during laboratory-based social stress. Neurosci Biobehav Rev. 2020;114:194-200. doi:10.1016/j.neubiorev.2020.04.005

36. Juster RP, Raymond C, Desrochers AB, et al. Sex hormones adjust "sex-specific" reactive and diurnal cortisol profiles. Psychoneuroendocrinology. 2016;63:282-290. doi:10.1016/j. psyneuen.2015.10.012 
37. Zheng JR, Huang ZR, Zhao JJ, et al. A study of psychometric properties, normative scores and factor structure of beck anxiety inventory chinese version. Chin J Clin Psychol. 2002;010(001):4-6.

38. Zheng Y, Wei L, Gao L, Zhang G, Wong C. Applicability of the Chinese Beck Depression Inventory. Compr Psychiatry. 1988;29 (5):484-489. doi:10.1016/0010-440X(88)90063-6

39. Huang L, Yang TZ, Ji ZM. Application of the positive and negative affect scale in Chinese. Chinese Mental Health J. 2003;17:54-56.

40. Xie YN. Preliminary study of the reliability and validity about the simplified coping style questionnaire. Chin J Clin Psychol. 1998;2:114-115.

41. Buchanan TW, Driscoll D, Mowrer SM, et al. Medial prefrontal cortex damage affects physiological and psychological stress responses differently in men and women. Psychoneuroendocrinology. 2010;35:56-66. doi:10.1016/j.psyneuen.2009.09.006
42. Childs E, Vicini LM, De Wit H. Responses to the Trier Social Stress Test (TSST) in single versus grouped participants. Psychophysiology. 2006;43:366-371. doi:10.1111/j.1469-8986.2006.00414.x

43. Kothgasssner OD, Felnhofer A, Hlavacs H, et al. Salivary cortisol and cardiovascular reactivity to a public speaking task in a virtual and real-life environment. Comput Hum Behav. 2016;62:124-135. doi:10.1016/j.chb.2016.03.081

44. Stalder T, Kirschbaum C, Kudielka BM, Adam EK, Clow A. Assessment of the cortisol awakening response: expert consensus guidelines. Psychoneuroendocrinology. 2016;63:414-432. doi:10.10 16/j.psyneuen.2015.10.010

\section{Publish your work in this journal}

Psychology Research and Behavior Management is an international, peer-reviewed, open access journal focusing on the science of psychology and its application in behavior management to develop improved outcomes in the clinical, educational, sports and business arenas. Specific topics covered in the journal include: Neuroscience, memory and decision making; Behavior modification and management; Clinical applications; Business and sports performance management; Social and developmental studies; Animal studies. The manuscript management system is completely online and includes a very quick and fair peer-review system, which is all easy to use. Visit http://www. dovepress.com/testimonials.php to read real quotes from published authors. 